\title{
Mass Customization of Deployable Origami-based Structures
}

\author{
Bojan Tepavčevićl, Vesna Stojaković ${ }^{2}$, Dejan Mitov ${ }^{3}$ \\ ${ }^{1,2,3}$ Faculty of Technical Sciences, University of Novi Sad \\ ${ }_{1,2,3}\{$ tepavcevicb|vesna100|dejan_mitov\}@uns.ac.rs
}

In this research we present a design for a mass customization online $3 D$ model for deployable emergency shelter that automatically provides drawings for CNC machines. The main motivation for such research has risen from a global need to provide emergency shelters for people affected by natural disaster. The model is designed to be a flat packable, mono-material based on a double corrugated folding pattern. Based on numerous functional, structural and fabrication constraints the presented model can provide a myriad of similar geometric forms that can reflect personal needs and can be used for different purposes.

Keywords: Mass customization, folding pattern, digital fabrication, emergency shelters

\section{INTRODUCTION}

Origami patterns have been used in the process of architectural design for many decades. Due to the applicability of paper models in design experiments as well as load-carrying capability of certain folding patterns, origami inspired folding structures have been widely applied to the design of architectural structures (Buri, and Weinand 2008; Šekularac et al, 2012; Tao Shen and Nagai 2017). Recently, advances in computational design fostered extensive research on the application of origami-based structures in architectural design (Buri and Weinand 2008), construction (Tromer and Krupna 2006), energy efficient design (Martinez-Martin and Thrall 2014) and manufacturing (Robeller and Weinand. 2016a; Robeller and Weinand. 2016b). Additionally, many societal challenges as well as sustainable approaches to design affect the increasing interest towards transformable folding structures. As opposed to conventional static, structurally stable origami-based struc- tures, deployable structures have the capacity to efficiently respond to different boundary conditions, environmental and climatic conditions, functional requirements, or emergency situations (Temmerman et al. 2014).

In this research we present an open model for mass customization of a deployable origami-based structure. While current research of deployable origami-based structures is mainly focused on its geometry (Demaine and Tachi, 2017; Cai, Deng, Xu and Feng 2015) or structural behaviour (Chudoba, van der Woerd, and Hegger 2014; Lee and Gattas, 2016), we investigate how advantages of such structures can be used for mass-customization for real-world situations through the application of an online configurable model. The main motivation for such research has risen from a global need to provide emergency shelters for people affected by natural disaster. We have designed a mass customization online configurable 3D model for deployable emergency shelters 
that can automatically provide a myriad of similar geometric forms, based on predefined design, functional and construction constraints.

This research has been motivated by the international workshop held at the University of Novi Sad, organized under the framework of United Impact Academic Program, Green Contributor organization (Canada), BNCA School of Architecture, (Pune, India) and Digital Design Center, Faculty of Technical Sciences, University of Novi Sad. The aim of the workshop was to design and create simple flat packable and floatable emergency shelters which could withstand the pressure during natural disasters, including floods.

\section{METHOD}

The mass customized model presented in this paper is based on the geometry of a reverse folded pattern (Buri and Weinand 2008), also known as double corrugated [Mitra 2009] or the rigid-foldable accordion pattern (Lee and Gattas, 2016). All models are designed to be flat packable, which means that they are made from flat material sheets. The three dimensional structures based on origami folding can be unfolded for the purpose of storage or transport which adds to the efficiency and applicability of the origami-based design. The design process is created in a parametric environment, which allows adjustment of the shape and size to the personal needs of anyone who uses the model configurator. The size and shape of the shelters as well as the distribution of openings are also stored in an online parametric model, allowing the user to automatically create the drawing for CNC machining according to their own need. While design and construction of emergency shelters are often considered for serial production, the presented mass customization tool enables user participation and a variety of design solutions with a low unit cost associated with mass production. CNC machines are used to create kerfs on the material which represent the folding lines. This allows the material to be easily folded in providing emergency shelter or unfolded to the flat panel according to the cur- rent needs.

The creation of a mass customization tool that automatically generates the drawings for $\mathrm{CNC}$ milling consists of 4 phases: 1) design of the parametric model based on the geometry of a reverse folded pattern, 2) design of an online configurable model based on the parametric model, 3) material tests with mock-ups, 4) digital fabrication of the prototype.

\section{Design of the Parametric Model}

In order to provide a suitable digital environment for the design of the parametric model, Rhinoceros 3D with its graphical algorithm editor Grasshopper is used. The model is completely based on a parametric algorithm, which means that there is no need for manual 3D modelling. The 3D model of origamibased shelter and unfolded model are generated automatically and the shape of the shelter is adjustable with parameter settings. The shape is defined by the closed outline which represents the cross section of the foldable structure and the angles which determine the corrugation of a double corrugated origami pattern in a longitudinal direction (Figure 1). The shape of the outline and the angles are controlled by the configurator.

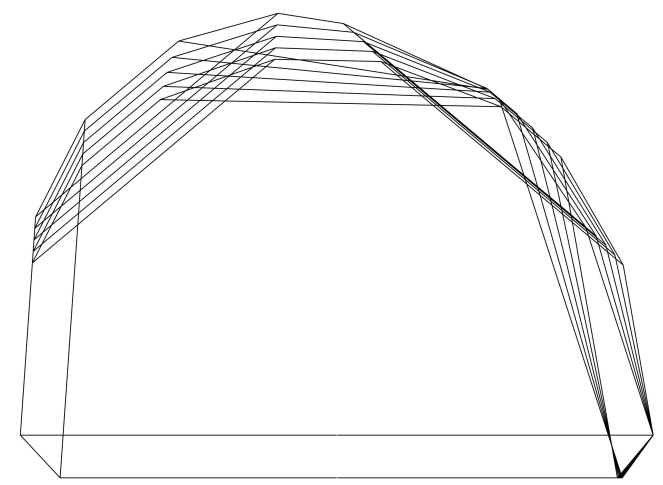

The first step towards the model design is creating a cross section outline. The outline is closed due to the project requirements for the structure to be floatable and stable without anchoring. The outline is a polyg-
Figure 1

Example of cross section shape variation controlled by a configurator parameter 
Figure 2

Geometry

Constraints for the

Cross Section

Outline onal line which has an even number of vertices. The number of vertices is important because each pair of neighbouring lines uses a different fold an inside and outside reverse fold. The fold changes from the inside fold to an outside fold and vice versa in each vertex.

Since the outline is closed, the even number of vertices is necessary in order to make a tunnel like reverse folded, closed structure. The outline starts with the horizontal line which is necessary because it represents the cross section of the floor of the structure. The shape of the outline is further specified by the parameters which determine the angles and the lengths for the rest of the lines. These parameters are adjustable by the user in a way that allows for the control of the resulting shape. The model is made to restrict the maximum perimeter of the polygon to be less than $3000 \mathrm{~mm}$ because the unfolded structure has to fit in the single sheet of material of that width. Additionally, the sum of polygon exterior angles is $360^{\circ}$ and the size of the angles are limited within the range defined by the perimeter of the polygon (Figure 2).

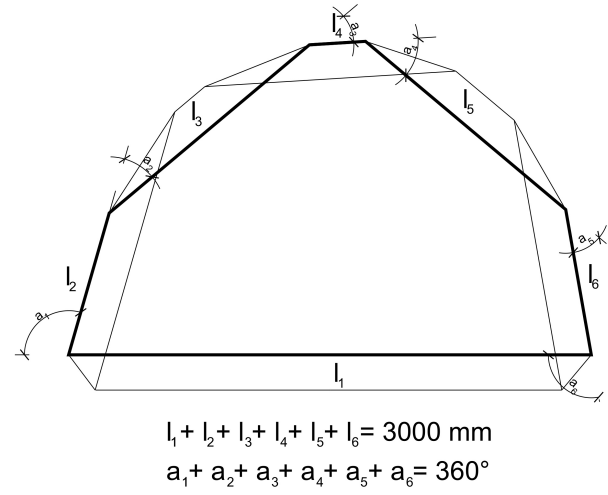

When the cross section outline is defined it is used to model the position of the ribs at each linear part of the outline. The slope of the ribs, defining the fold angle can also be controlled by the user.

After the slopes of a single rib for each line is defined, the ribs are multiplied creating the corrugated origami-based tree dimensional structure. The total number of ribs can be selected by the user. However, the parameter which controls the number of ribs is constrained to a set maximal length of the unfolded structure to be less than $1500 \mathrm{~mm}$ in order to fit in one sheet of the material of that length.

When all the parameters are set the origamibased three dimensional structures are created. The structure's unfolding mechanism is also automatized. That means that the user defines what he desires to create a 3D and technical 2D drawing of an unfolded structure (which can be directly used for CNC cutting) and is automatically generated according to the parameters entered.

\section{Design of an Online Configurable Model}

After the parameters and workflow for the model are designed, an online configurable model based on the parametric model is created. The model is exported online by the ShapeDiver add-on for Grasshopper3D. Anonline environment for this parametric model allows a wider audience to easily approach the configurators and use the algorithm. Users that want to design and fabricate their own emergency shelter need only a smartphone and internet connection to do it. The advantage of such a configurator is that no special CAD or 3D modelling software has to be installed on the computer and no previous knowledge about 3D modelling or parametric modelling is required in order to send necessary inputs to the CNC machine and manufacturer. In addition the online configurator has only controllable parameters and the viewport showing the 3D model and unfolded pattern which is generated according to these parameters. Likewise the shape parameters are constrained to ensure that the 2D unfold can fit a single sheet of material. Then the export button enables sending a dwg file format ready for the milling process. This process of parametric modelling of the tree dimensional structure and unfolding of the structure is done in the background. The application of ShapeDiver add-on enables an iframe embedding link that can be copy -pasted directly in any 


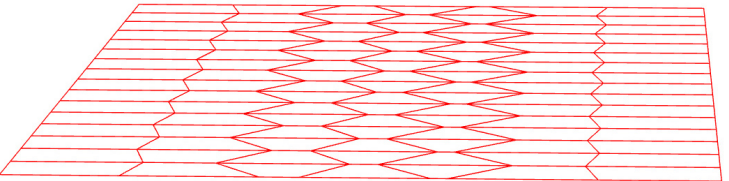

§ั

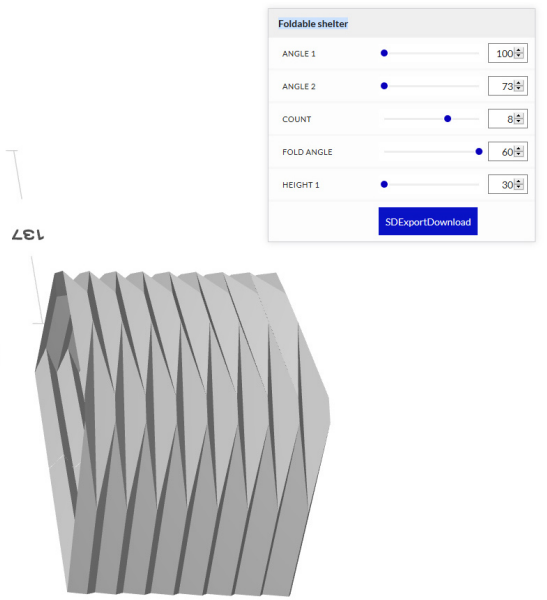

Figure 3

Interface Design of

Parametric 3d

Configurator for the

Emergency Shelter webpage. Additional options including managing a camera or light sources in a 3D scene is accessible through ShapeDiver's API, which is based on Tree.js WebGL based library. The online model presented in this work (Figure 3) can be accessed on the following link: www.arhns.uns.ac.rs/cdd/foldable-shelters

\section{Material Tests with Mock-ups}

The material tests with the mock-ups are made in order to test the foldability, which means finding a suitable thickness of the material, as well as the width and depth of the kerf. Apart from the common material applied for emergency shelters that utilize corrugated cardboard or fabric, for this research a polyethylene PE-500 $3 \mathrm{~mm}$ thick sheet was used. PE-500 is a material with good physical and mechanical properties for creating mono-material deployable shelters. PE-500 is a non-hygroscopic environmentally friendly material, which exhibits high impact resistance and excellent machinability. A good combination of stiffness and toughness enables the PE500 material sheets to fold multiple times along the kerfs without fracturing. For testing foldability of PE500 sheets, we folded mock-ups 20 times from the unfolded planar stage, to the maximum folding po- sition. Due to the high level of material toughness PE-500 sheet mock-ups showed that it can be used multiple times without cracks.

We made tests on 3 mock-ups to find a suitable kerf width, depth and orientation (Figure 4). We started with the presumption that the material had to be kerfed from both front and back side, in order to enable reverse folding easy. However, kerfing on both sides of the material created holes in the places of intersection on both frontside and backside kerf lines (Figure 4 left). For this reason we created two additional tests with kerf lines only on one side of the material to find an optimal kerf size and concluded that $2.3 \mathrm{~mm}$ deep kerfs are suitable for fabrication on $3 \mathrm{~mm}$ thick PE-500 sheets (Figure 4 middle). Additionally, kerf width of $10 \mathrm{~mm}$ is suitable comparing to depth because it is large enough to enable $180^{\circ}$ folding without weakening the material (Figure 4 right).

\section{Digital fabrication of the Prototype}

The dimension of the polyethylene PE - 500 sheet we used was $3000 \times 1500 \mathrm{mmm}$ and the prototype was manufactured in a 1:2 scale. The size of the shelter can be larger if the larger sheet is used or if the two or more sheets are connected. Polyethylene sheets 
Figure 4

Material Tests with Mock-Ups

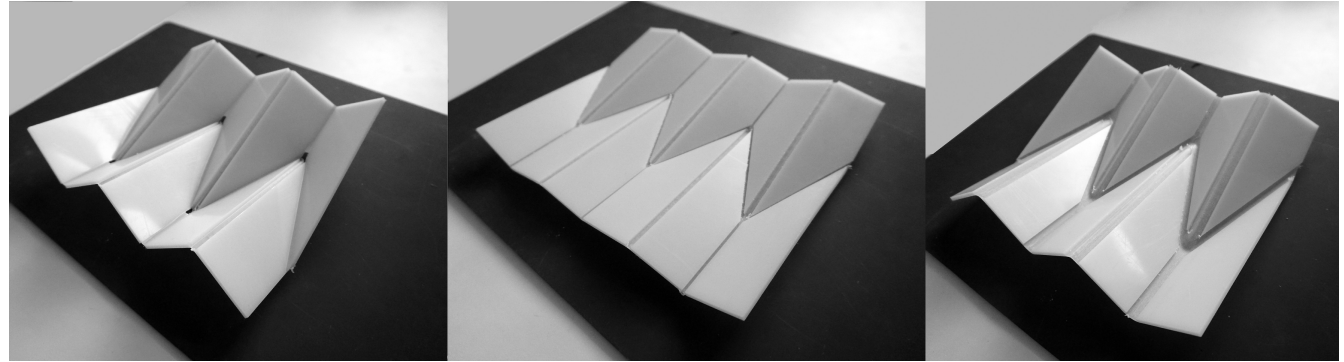

can be connected with a puzzle joint connection or by welding process. This puzzle joint connection is based on friction-fit assembly logic which can provide making large structures at low unit cost from small sheets of the material without any additional tools or adhesives (Tepavčević et al 2017; Sass and Botha 2006). Similar properties of the material and kerfing can be used for the full scale emergency shelter prototype.

Kerfs are made by using a CNC milling machine. Since the kerfs allow $180^{\circ}$ folding, the prototype can be folded by any fold angle during the assembly without the risk of material breaking. This is important because it would be impossible to fold the material only up to a particular angle during the assembly. For each shelter one piece of the material was used, so there was no stitching of the material during the assembly, only folding (Figure 5). When folded, the prototype had to be glued only on the upper side to create a continuous structure.

\section{DISCUSSION}

In this research we focused on the process of creating a mass customization model which can be used in the design-to-fabrication process for deployable origami-based emergency shelters. Compared to existing solutions, we decided to focus on creating emergency shelters that can be personalized through a mass customization process and yet costefficient, easy to use, pack or transport. In order to satisfy all this criteria we focused on mono-material folding solutions. The geometry of a reverse folded pattern used in the creation of this parametric model has many advantages in the context of their use as emergency shelters. A cross-section of the shape is a closed polygon generating a structurally stable origami-based structure.

A reverse folded pattern configuration consists of planar trapezoids. Compared to the triangular structure, it has only four planes that intersects at one common intersection point. Due to the lower number of planes that intersects at a common point, the assembly process is much easier.

Many origami-based emergency shelters are manufactured with cardboard material $[1,2]$. Cardboard origami shelters are also a sustainable and compact mono-material shelter as the presented one, based on PE-500. However, cardboard shelters need waterproof coating in case of its usage against the rain. Resistance of waterproof coating is not fully reliable in cases of devastating monsoon floods. Using a polyethylene PE-500 not only provides protection from rain, but can also provide a design based on buoyancy principles.

Finally, we show that design of such a structure can be distributed to end users with a web page link, providing instant design as well as variability and adjustability to the specific needs and number of users. 


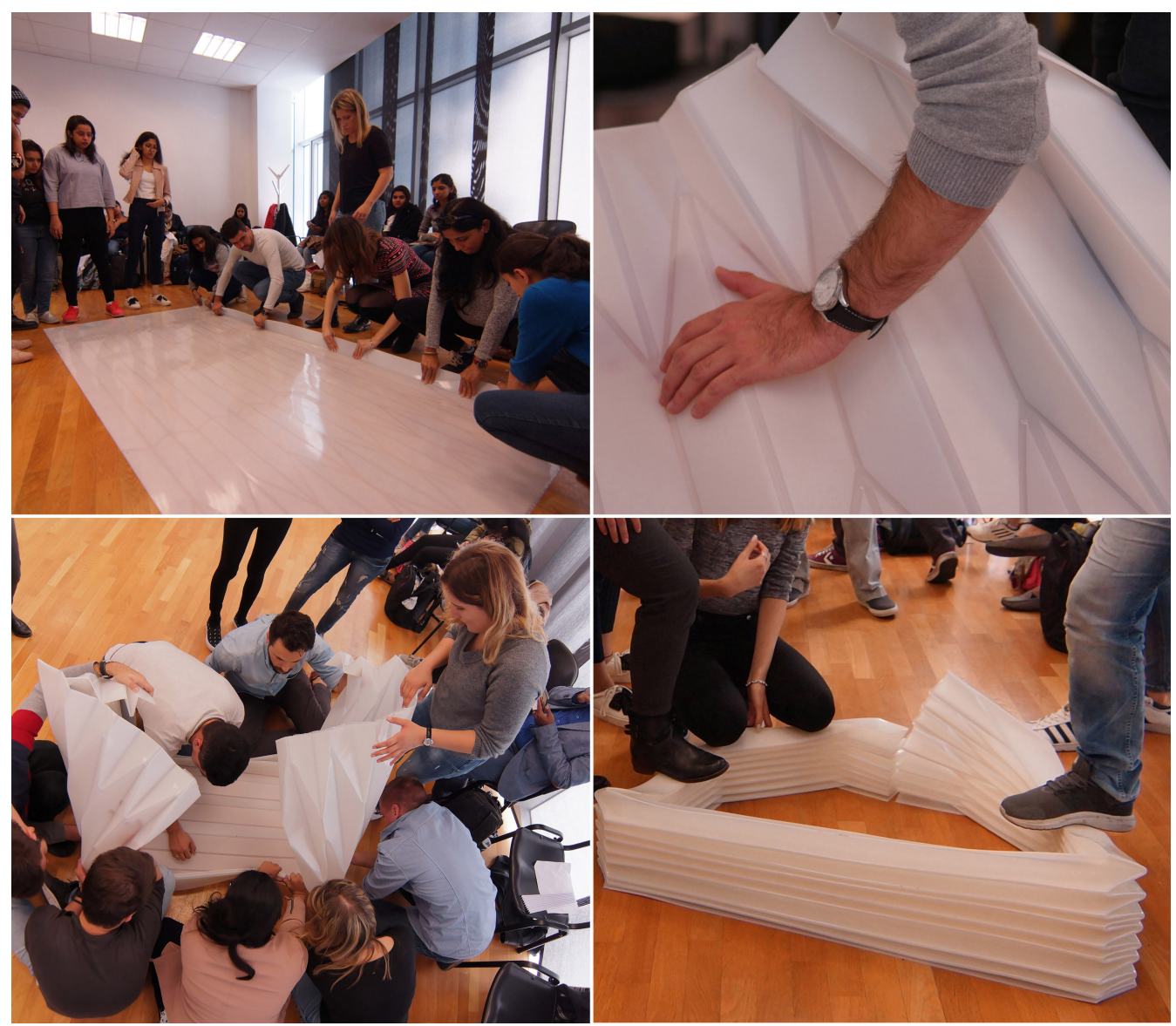

Figure 5

Folding Process 
Figure 6

Buoyancy Test

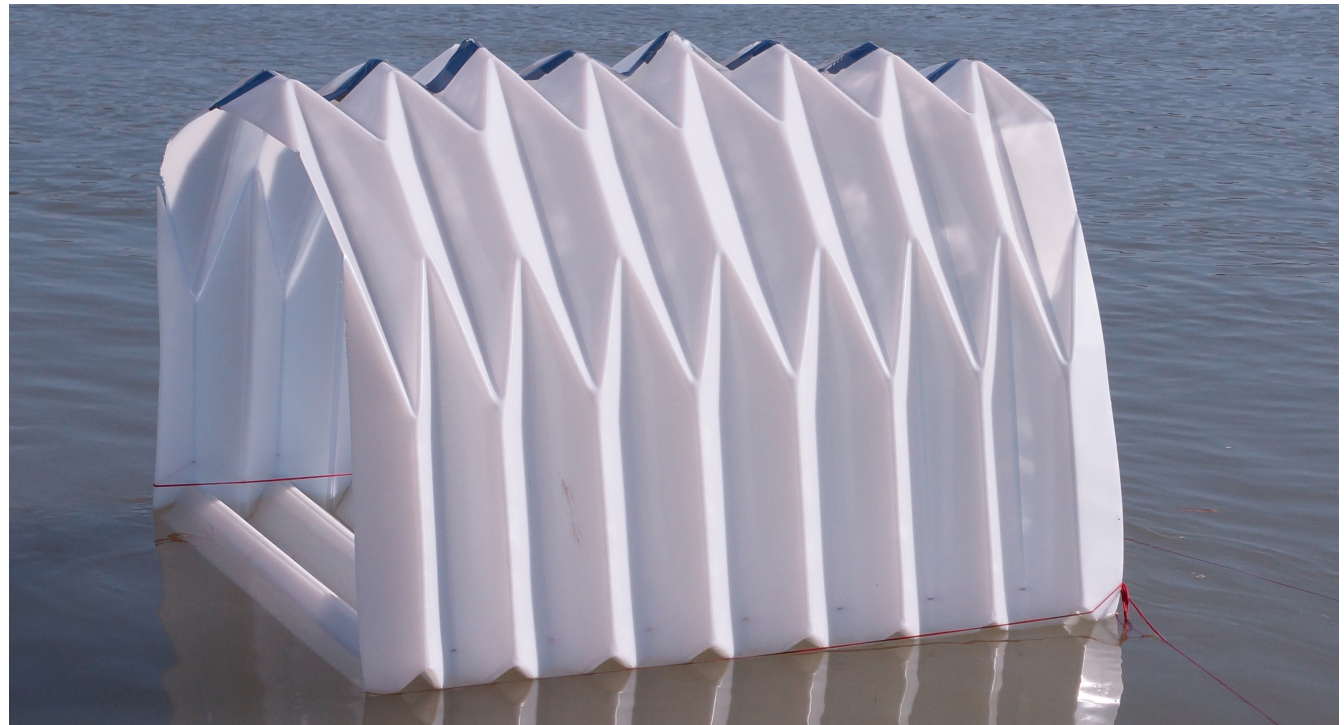

\section{CONCLUSION}

The mass customisation model shows that due to numerous functional, structural and fabrication constraints, the origami patterns based on reverse folding provide a solid base for creating a myriad of forms for shelters that can reflect personal needs and be used for different purposes. An origami based design made only by folding from a single piece of flat material used in this paper demonstrates that it is possible to create the tree dimensional structure that can be easily folded and unfolded depending on current need. This is an important benefit for an emergency shelter which is a temporary structure since it can be efficiently stored and easily transported onsite.

The mass customisation tools are useful if they are available to a broad audience. Transferring the 3D parametric model to an online platform in order to generate the model and cutting scheme for a CNC machine demonstrates the full potential of mass customization.

\section{ACKNOWLEDGEMENTS}

This research was supported by the Serbian Ministry of Education and Science under contracts TR36042 and OI 174012. The authors also appreciative of the invaluable help in organizing workshop to professors Asmita Joshi, Dhanashree Sardeshpande BNCA Pune, India and George Koshy, representative of the green contributor organization, Canada. Thank you also to the students who participated in the workshop.

\section{REFERENCES}

Buri, H and Weinand, Y 2008 'Origami- Folded Plate Structures, Architecture', 10th World Conference on Timber Engineering, Miyazaki

Cai, J, Deng, X, Xu, Y and Feng, J 2015, 'Geometry and Motion Analysis of Origami-Based Deployable Shelter Structures', Journal of Structural Engineering, 141(10), p. 06015001

Chudoba, R, van der Woerd, J and Hegger, J 2014, 'Numerical Modeling Support for Form-Finding and Manufacturing of Folded-Plate Structures Made of Cementitious Composites Using Origami Principles', in Bićanić, N, Mang, H, Meschke, G and de Borst, $\mathrm{R}$ (eds) 2014, Computational Modelling of Concrete 
Structures, Taylor and Francis group, London, pp. 451-461

Demaine, E and Tachi, T 2017 'Origamizer: A Practical Algorithm for Folding Any Polyhedron', The 33rd International Symposium on Computational Geometry, Brisbane, Australia

Lee, T and Gattas, J 2016, 'Geometric, Design and Construction of Structurally Stabilized Accordion Shelters', J. Mechanisms Robotics, 8(3), pp. 031009-1031009-8

Martinez-Martin, F.J and Thrall, A.P 2014, 'Honeycomb Core Sandwich Panels for Origami-Inspired Deployable Shelters: Multi-Objective Optimization for Minimum Weight and Maximum Energy Efficiency', Engineering Structures, 69, p. 158-167

Mitra, A 2009, The Grammar of Developable Double Corrugations (for Formal Architectural Applications), Ph.D. Thesis, University College London

Robeller, C and Weinand, Y 2016a, 'Integral Joints for Timber Folded Plate Structures', in Menges, A, Schwinn, T and Krieg, O.D. (eds) 2016a, Advancing Wood Architecture - A Computational Approach, Routledge, London, pp. 73-84

Robeller, C and Weinand, Y 2016b, 'Fabrication-Aware Design of Timber Folded Plate Shells with Double Through Tenon Joints', in Reinhardt, D, Saunders, R and Burry, J (eds) 2016b, Robotic Fabrication in Architecture, Art and Design, Springer, Cham, p. 166-77

Sass, L and Botha, M 2006, 'The instant house: a model of design production with digital fabrication', International Journal of Architectural Computing, 4(4), p. 109-123

Shen, T and Nagai, Y 2017, 'An Overview of Folding Techniques in Architecture Design', World Journal of Engineering and Technology, 5, pp. 12-19

De Temmerman, N, Roovers, K, Mira, L.A, Vergauwen, A, Koumar, A, Brancart, S, Laet, L.D and Mollaert, M 2014, 'Lightweight transformable structures: materialising the synergy between architectural and structural engineering', WIT Transactions on The Built Environment, 136, pp. 1-20

Tepavčević, B, Stojaković, V, Mitov, D, Bajšanski, I and Jovanović, M 2017, 'Design to fabrication method of thin shell structures based on a friction-fit connection system', Automation in Construction, 84, pp. 207-213

Trometer, S and Krupna, M 2006, 'Development and Design og Glass Folded Plate Structures', Journal of the International Association for Shell and Spatial Structures, 47(3), pp. 1-20
Šekularac, N, Ivanović, S.J. and Čikić, T.J. 2012, 'Folded Structures in Modern Architecture', Facta Universitatis, Series: Architecture and Civil Engineering, 10(1), p. $1-16$

[1] https://joegattas.com/plate-house/

[2] https://www.cardborigami.org/ 